\title{
Incidental findings suggestive of COVID-19 in asymptomatic cancer patients undergoing 18F-FDG PET/CT in a low prevalence region
}

\author{
Amandine Pallardy ${ }^{1}$ (1) $\cdot$ Caroline Rousseau $^{2,3} \cdot$ Catherine Labbe $^{4} \cdot$ Renan Liberge $^{5} \cdot$ Caroline Bodet-Milin $^{1,3}$. \\ Françoise Kraeber-Bodere ${ }^{1,2,3} \cdot$ Vincent Fleury $^{2}$
}

Received: 26 May 2020 / Accepted: 24 August 2020 / Published online: 28 August 2020

(C) Springer-Verlag GmbH Germany, part of Springer Nature 2020

\begin{abstract}
Purpose To assess the rate and pattern of incidental interstitial lung abnormalities suggestive of COVID-19 on 18F-FDG PET/ CT in asymptomatic cancer patients during the period of active COVID-19 circulation between March and April 2020 in a geographic area of low prevalence of the virus.

Methods 1396 18F-FDG PET/CT performed between January 1, 2020, and February 21, 2020, and between March 16, 2020, and April 17, 2020 for routine oncological indication were retrospectively analyzed. No patients had symptoms suggestive of COVID-19 at the time of the 18F-FDG PET/CT. Incidental interstitial pneumonias suggestive of COVID-19 were identified, and the 18F-FDG PET/CT patterns were described. We compared the incidence of these lesions in the pre-COVID and pandemic phases.

Results We observed a $1.6 \%$ increase in interstitial lung abnormalities during the period of COVID-19 circulation. All had $<50 \%$ lung involvement. We describe a case series with typical and atypical interstitial pneumonias suggestive of COVID-19 as unilateral or bilateral with ground-glass opacity, consolidation, or crazy-paving patterns.

Conclusion The relatively low increase in incidental findings suggestive of COVID-19 infection on 18F-FDG PET/CT in asymptomatic cancer patients was in accordance with the low COVID-19 transmission in our geographic region. Nevertheless, nuclear medicine physicians should familiarize themselves with typical and atypical 18F-FDG PET/CT patterns suggestive of COVID-19 pneumonia and initiate appropriate intervention where necessary.
\end{abstract}

Keywords PET/CT $\cdot 18 \mathrm{~F}-\mathrm{FDG} \cdot$ Lung $\cdot$ Infection $\cdot$ Covid-19

This article is part of the Topical Collection on Infection and inflammation

Amandine Pallardy

amandine.pallardy@chu-nantes.fr

1 Nuclear Medicine Department, University Hospital of Nantes, Nantes, France

2 Nuclear Medicine Department, Institut de Cancérologie de l'Ouest René Gauducheau, Saint-Herblain, France

3 CRCINA, INSERM, CNRS, Université d'Angers, Université de Nantes, Nantes, France

4 Radiology, Institut de Cancérologie de l'Ouest René Gauducheau, Saint-Herblain, France

5 Radiology, University Hospital of Nantes, Nantes, France

\section{Introduction}

In December 2019, the novel coronavirus disease 19 (COVID-19) emerged in Wuhan, China. The virus (SARSCoV-2) spread rapidly around the globe and was declared a pandemic in March 2020 by the World Health Organization.

The COVID-19 clinical symptoms are well-known, and the radiological lung imaging were features widely described [1]. Chest CT imaging of confirmed COVID-19 infection mainly shows typical peripheral or subpleural ground-glass opacities (GGO) and ground glass with consolidation and crazy paving $[1,2]$.

Some patients may be asymptomatic at the beginning of the infection or for the entire period of the disease and may contribute to the diffusion of the virus among the population [1]. Chest CT can identify signs of COVID-19 in asymptomatic subjects [3]. In asymptomatic subjects, radiological abnormalities are more likely to be unilateral, multifocal, predominantly GGOs that will rapidly evolve to bilateral disease [4]. 
Patients with cancer who are referred to a nuclear medicine department for 18F-FDG-PET/CT screening may have previously contracted COVID-19 but remain asymptomatic. Incidental findings suggestive of COVID-19 may not be infrequent in hybrid imaging of asymptomatic patients, in geographic regions with expansive spread of COVID-19 infection [5].

The aim of this 18 F-FDG-PET/CT case series is to compare the incidence of these incidental findings in the pre-COVID period to those in the pandemic phase and to describe various suspicious presentations with COVID-19 incidental findings in asymptomatic patients in an area of low prevalence of the virus.

\section{Materials and methods}

\section{Population}

We retrospectively reviewed PET/CT scans performed on COVID-19 asymptomatic cancer patients for routine oncological indication in the Nantes PET Center France, from January 1, 2020, to February 21, $2020(n=867$ PET/CT) and from March 16, 2020, to April 17, 2020 $(n=529 \mathrm{PET} / \mathrm{CT})$.

The period from March 16, 2020, to April 17, 2020, was the beginning of the period of social containment in France, and patients were determined to be asymptomatic for COVID19 infection following questioning and systematic facial temperature measurements before entering the Nuclear Medicine Department. The period from January 1, 2020, to February 21 is considered to be the period before defined COVID-19 epidemia in our region.

Patients younger than 18 years old and patients with concomitant known inflammatory lung diseases were excluded from our report. No suspicion of viral infection was present in the days before or at the time of the PET/CT scan in any of our patients.

\section{PET/CT protocols}

Oncological 18F-FDG PET/CT were performed on patients fasted for at least $6 \mathrm{~h}$, with blood glucose < $12 \mathrm{mmol} / \mathrm{L}, 60 \mathrm{~min}$ after injection of $3 \mathrm{MBq} / \mathrm{kg}$ of $18 \mathrm{~F}-$ FDG, with a free-breathing helical CT, using either a Biograph mCT64 or a Vision 450 PET/CT (Siemens). An additional deep breath $\mathrm{CT}$ scan was performed if there was any doubt about $\mathrm{CT}$ abnormalities.

All PET/CT were reviewed by two experienced nuclear medicine physicians specializing in thoracic imaging. In case of doubt, a review was carried out by a radiologist. The criteria used were the presence of GGOs, consolidation, crazy paving, peripheral or central distribution, number of lobes involved, and unilateral or bilateral distribution. Visual quantification of lung involvement was classified as minimal if less than $10 \%$, moderate between 10 and $25 \%$, and significant between 26 and $50 \%$ as recommended by the French Thoracic Imaging Society. Maximum standardized uptake value (SUVmax) measurements were reported.

\section{Results}

Before the outbreak of COVID-19 (Jan 2020 to Feb 2020 cohort), CT abnormalities consistent with interstitial lung disease were found in $2.2 \%$ of patients $(n=19) .17 / 19$ of these lesions were $18 \mathrm{~F}-\mathrm{FDG}$ positive (range SUVmax from 2.23 to 12.05).

During the containment period (March 2020 to April 2020), CT interstitial anomalies were identified in $3.8 \%(n=$ 20 ) of patients. $18 \mathrm{~F}-\mathrm{FDG}$ avidity was observed in 18 out of 20 patients with a SUVmax ranging from 1.58 to 13.64.

A pattern of unilateral abnormality was observed in 12 patients $(60 \%$, case 1$)$. A bilateral abnormality was observed in 8 patients $(40 \%)$. Of these, 2 had minimal involvement (case 2), 4 had moderate involvement (case 3), and 2 had significant involvement (case 4). We did not observe more than $50 \%$ involvement in our cohort. Case 5 also illustrated the nonspecific crazy-paving pattern.

\section{Case 1}

A 63-year-old woman underwent 18F-FDG PET/CT for staging of locally advanced triple negative breast cancer. PET/CT showed a FDG-avid left breast mass and positive axillary lymph nodes. Additionally, focal GGOs with partial consolidation, ill-defined, and subpleural distribution in the middle lobe with FDG uptake were visualized (Fig. 1).

\section{Case 2}

A 57-year-old woman diagnosed with lymph node metastatic breast cancer underwent an 18F-FDG PET/CT for postoperative staging. PET/CT showed GGOs with increased FDG uptake in the lower right lobe and subpleural GGOs in the upper left lobe without FDG uptake. Bilateral lung opacities were less than $10 \%$ of the lung parenchyma (Fig. 2).

\section{Case 3}

A 70-year-old man underwent 18F-FDG PET/CT for the assessment of a lung nodule. PET/CT performed 1 month after chest $\mathrm{CT}$ showed new GGOs and consolidation of the 5 lobes with peripheral and lower lobe predominance with FDG uptake. Bilateral lung involvement was moderate. The rapid 
Fig. 1 Maximum intensity projection (MIP) (a) showing hypermetabolic left breast mass and axillary lymph nodes (red circle). Subsequent axial CT (b), PET (c), and fused PET/CT images (d) showing focal GGOs with partial consolidation in the middle lobe, with moderate 18 F-FDG uptake (orange arrows). SUVmax 3.06

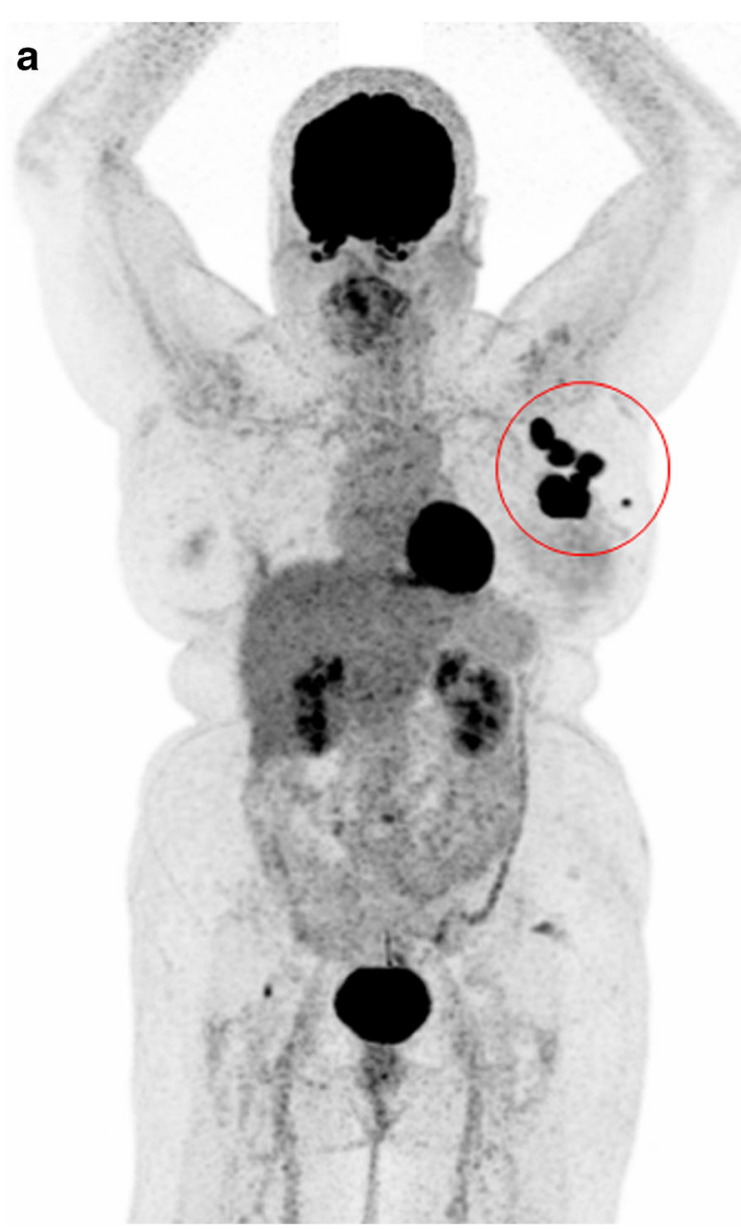

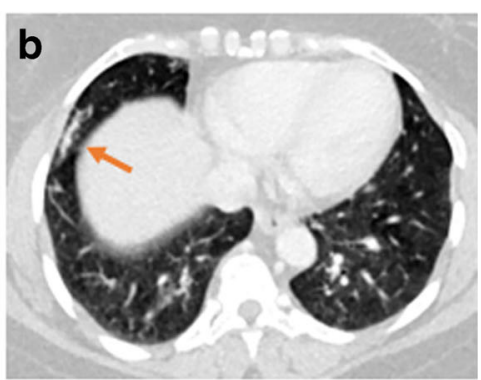
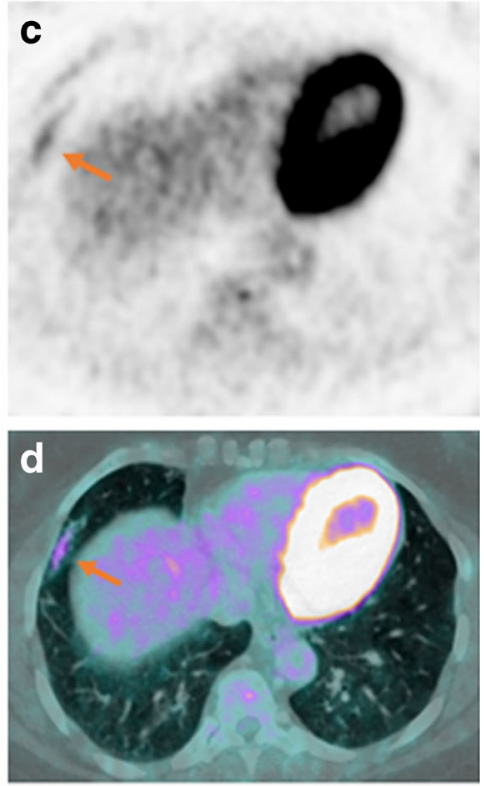

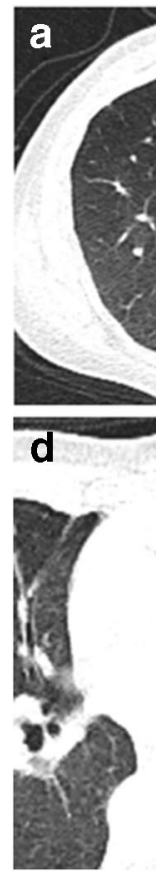

b
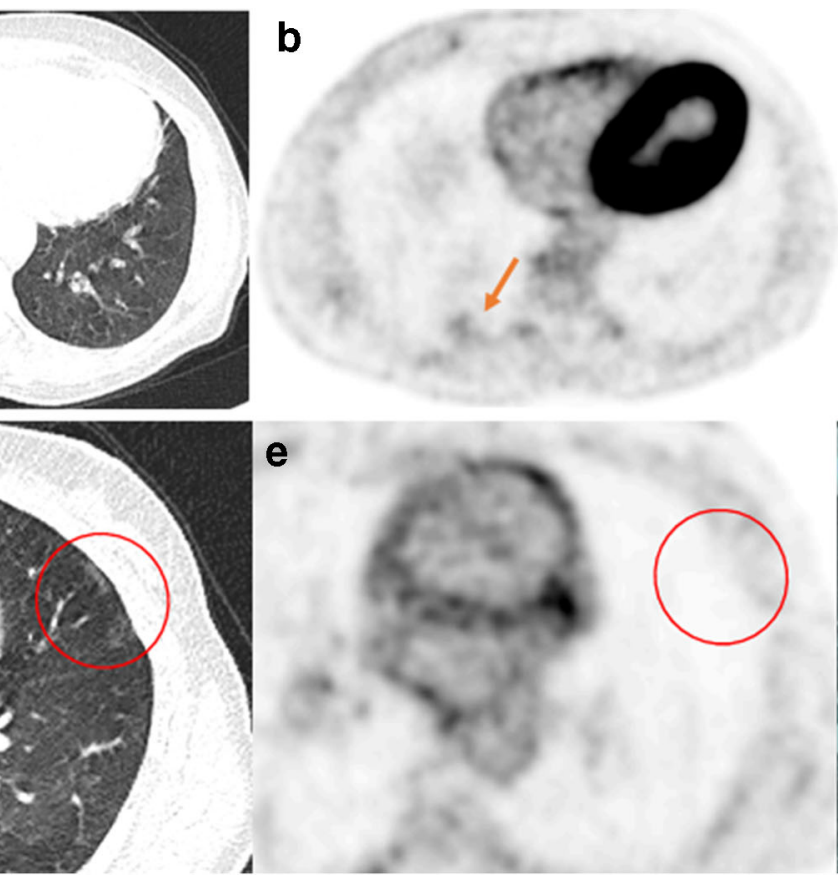
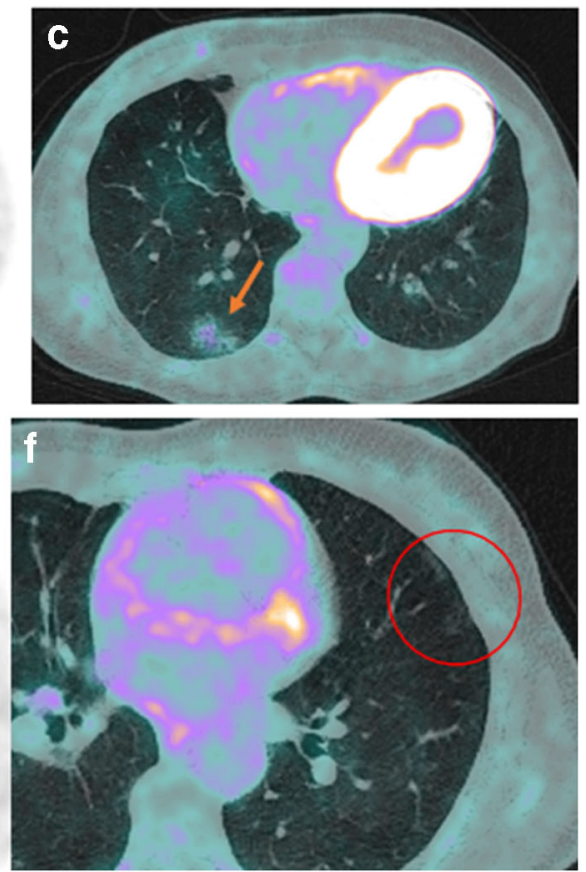

Fig. 2 Subsequent axial CT $(\mathbf{a}, \mathbf{d})$, PET (b, e), and fused PET/CT images (c, f) PET/CT showing GGOs with moderate 18F-FDG uptake in the lower right lobe (SUVmax 2.95) (orange arrows) and GGOs subpleural in the upper left lobe without 18F-FDG uptake (red circle) 
evolution of lung opacities and no symptoms were suggestive of COVID-19 (Fig. 3).

\section{Case 4}

A 65-year-old woman affected by metastatic breast cancer was referred for $18 \mathrm{~F}-\mathrm{FDG} \mathrm{PET} / \mathrm{CT}$ to assess therapy response. When compared with her previous exam, PET/CT FDG showed a lower uptake in the mediastinal nodes, contrasting with the appearance of bilateral and peripheral GGOs and consolidation with FDG uptake in the 5 lobes. The appearance of these lung opacities without symptoms was strongly indicative of COVID-19 in the context of a pandemic (Fig. 4).

\section{Case 5}

A 63-year-old man was referred for 18F-FDG PET/CT for staging of left lung adenocarcinoma. PET/CT confirmed increased FDG uptake of the lung mass and showed minimal FDG uptake of diffuse GGO with superimposed interlobular septal thickening and intralobular lines mostly in the left upper lobe of the lung. This crazy-paving pattern offers a wide range of differential diagnoses, in this context bronchioloalveolar carcinoma or intra-alveolar hemorrhage, and is not specific of COVID-19 infection (Fig. 5).

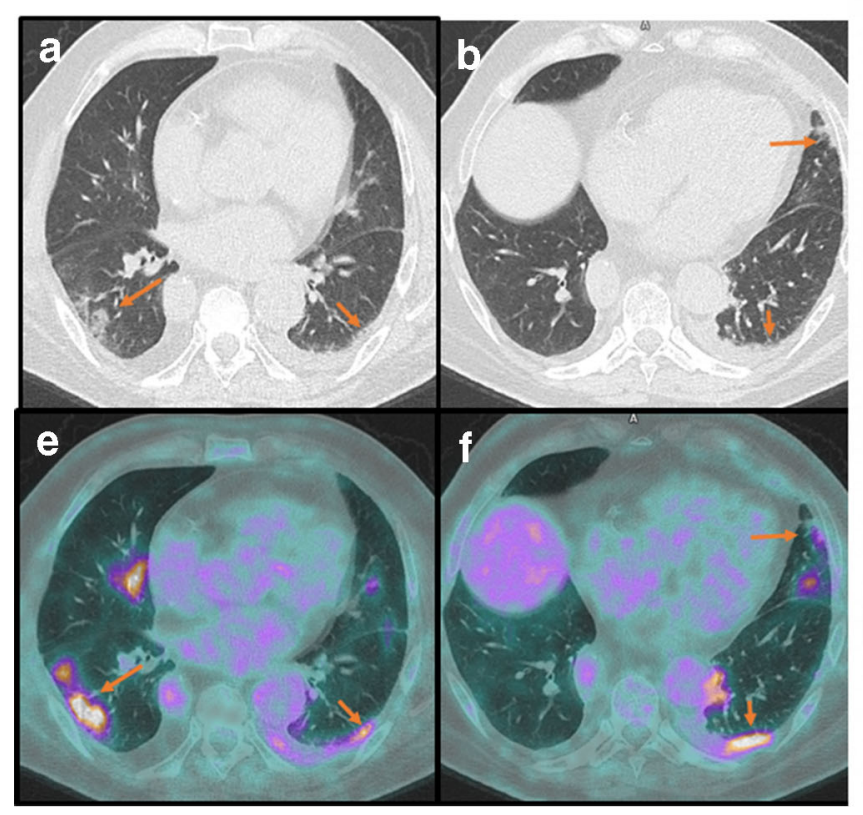

Fig. 3 Subsequent axial CT $(\mathbf{a}, \mathbf{b})$ and fused PET/CT images $(\mathbf{e}, \mathbf{f})$ showing ground-glass opacities in the periphery of the lungs with high $18 \mathrm{~F}$ FDG uptake (orange arrows). SUVmax 13.64. MIP (c) demonstrating moderate bilateral lung involvement. Additional deep breath CT images (d) allows to improve visualization of ground-glass opacities in the right

\section{Discussion}

We showed an increase of $1.6 \%$ in lung opacities suspected of COVID-19 in asymptomatic patients during the containment period. Although this appears to be a small increase, it is of the same order as the rate of circulation of SARS-CoV-2 in our region (the cumulative rate of patients tested positive for COVID-19 in our department was 59.1 per 100,000 inhabitants on April 17, 2020, according to Agence Régionale de Santé Pays de Loire). This population must be considered a group of risk, due to several reasons in particular because of the immunosuppression that may be induced by disease or ongoing therapies.

The typical pattern of $\mathrm{CT}$ imaging features for COVID19 is well-known with GGOs and/or consolidation, most often bilateral, rather peripheral and with lower lobe involvement [6]. Here, we report various forms of suspected presentations of COVID-19 in asymptomatic patients. Unilateral involvement is not uncommon [3, 4], up to $60 \%$ in subclinical patients [4]. Our findings concur, but false positive cases are possible. In addition, the extent of CT abnormalities is usually minimal to moderate in these patients, but significant involvement, more than $25 \%$ of the lung parenchyma, can be observed even without symptoms.

The CT parameters of PET/CT are not as efficient as those of diagnostic chest CT scans. However, in case of doubt, we
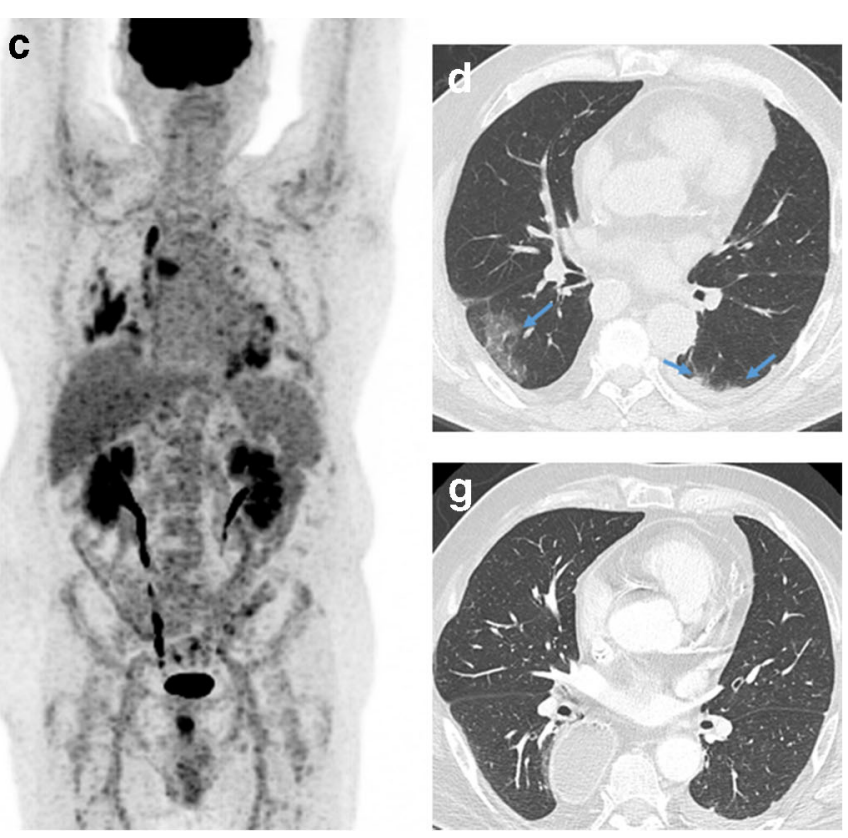

lower lobe and left lower lobe in contact with a pleural effusion without 18F-FDG uptake (blue arrows). Previous CT thorax axial images (g) show that the pleural effusion is identical and ground-glass opacities were not present 
have made an additional deep breath $\mathrm{CT}$ in order to limit respiratory artifacts.

In our study, the majority of patients with suspected COVID were FDG-positive [5, 7]. However, FDG-negative abnormalities such as curvilinear subpleural opacities remain suspicious for COVID-19.

The main limitation of our study is the lack of real-time reverse transcriptase polymerase chain reaction (rRTPCR) confirmation of FDG PET/CT cases, because at that time of the outbreak, the regional health and government authorities in our country do not recommend RT-PCR tests in the absence of symptoms. In our series, only 4 patients had an RT-PCR test after PET/CT. Two patients had significant lung involvement. For one, nasopharyngeal RT-PCR was positive. For the other, RT-PCR was negative. This is a patient being treated with ribociclibfulvestrant for breast cancer. Drug-induced lung disease with this treatment is very rare. Ribociclib can induce dyspnea and cough, but this patient was not symptomatic. Due to the context, clinical presentation, and sensitivity of RT-PCR, a negative test does not eliminate the diagnosis of COVID-19. The two others patients were addressed for staging of cancer, and they had minimal and moderate unilateral involvement. Nasopharyngeal RT-PCR tests were negative for both, and one had a PCR test bronchoalveolar lavage fluid because of development of respiratory symptoms a few days after PET/CT which was also negative for COVID-19. For these patients, clinical evolution was favorable.

In the other patients, in the absence of RT-PCR tests, given the overlap of CT and 18F-FDG PET/CT imaging features between COVID-19 and another interstitial lung disease like other viral pneumonia or cancer therapyinduced lung disease, false-positive cases of COVID-19 can be identified. Aware that imaging is not recommended as a screening tool for COVID-19 and more specifically FDG PET/CT. However, in the event of findings suggestive of COVID-19, measures must be taken to limit the spread. For all patient with suspicion of COVID-19, we informed them and advised to respect social distancing measures and barrier gestures. We have also informed the patient's medical referent in order to set up surveillance in these patients considered a group of risk.

Because of the lack of consensus in France for the treatment of asymptomatic patients, no specific treatment has been received.

Another limitation is the comparison with a control group considered to be free from risk of COVID-19 infection. In some parts of Europe in that period, the viral infection was already present. This could constitute a bias.

When available, serological tests could be proposed to refine and retrospectively validate the COVID-19 lung infection pattern; but they are not currently fully validated in France.
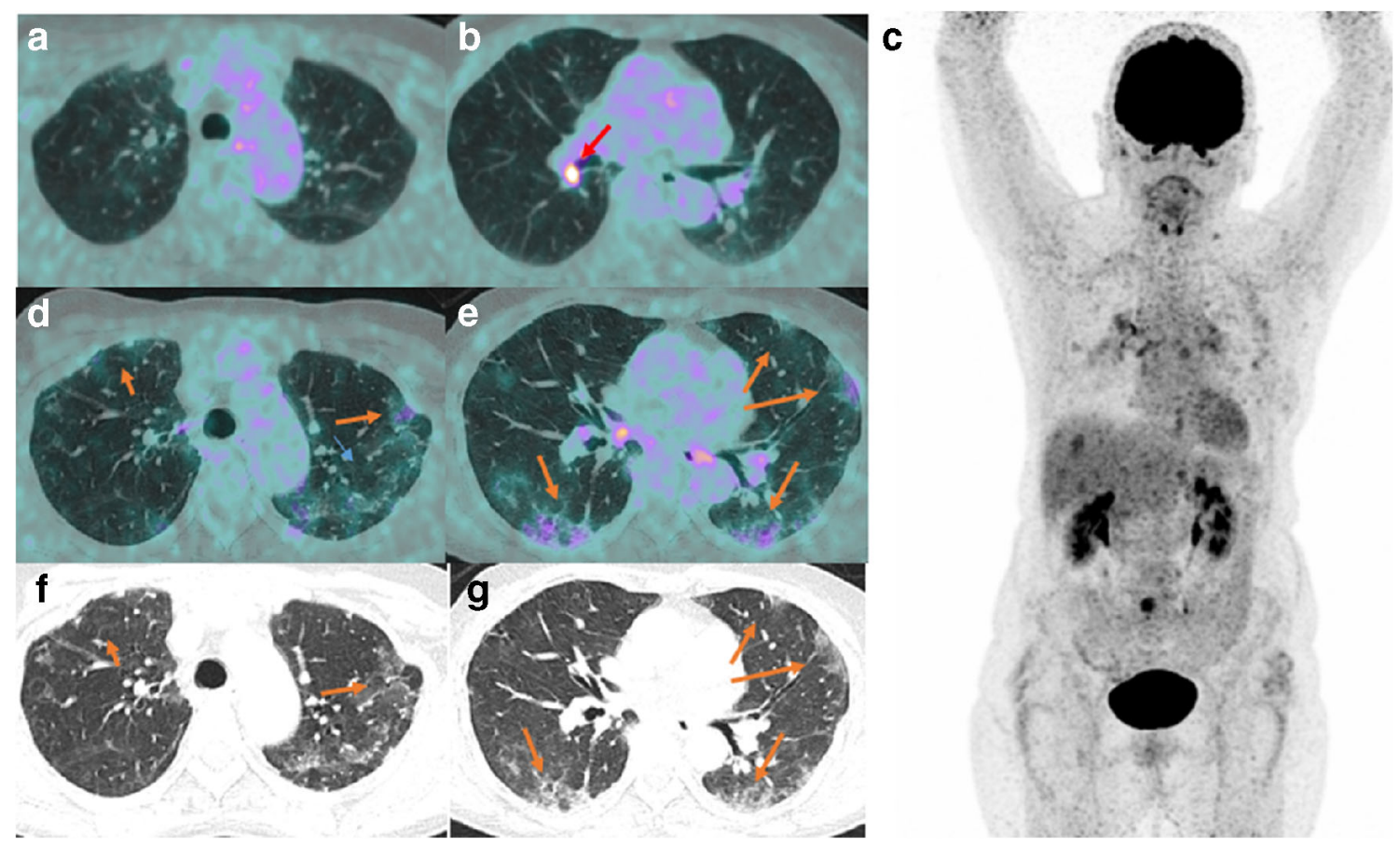

Fig. 4 Subsequent axial of previous fused PET/CT images $(\mathbf{a}, \mathbf{b})$ showing lymph node metastasis of breast cancer in the right hilum with avid $18 \mathrm{~F}$ FDG (red arrow). Subsequent fused PET/CT (d, e) and axial CT images

(f, g) showing a decrease in 18F-FDG uptake in the right hilum and new bilateral and peripheral GGOs and consolidation with moderate $18 \mathrm{~F}-\mathrm{FDG}$ uptake (orange arrows). SUVmax 4.53 


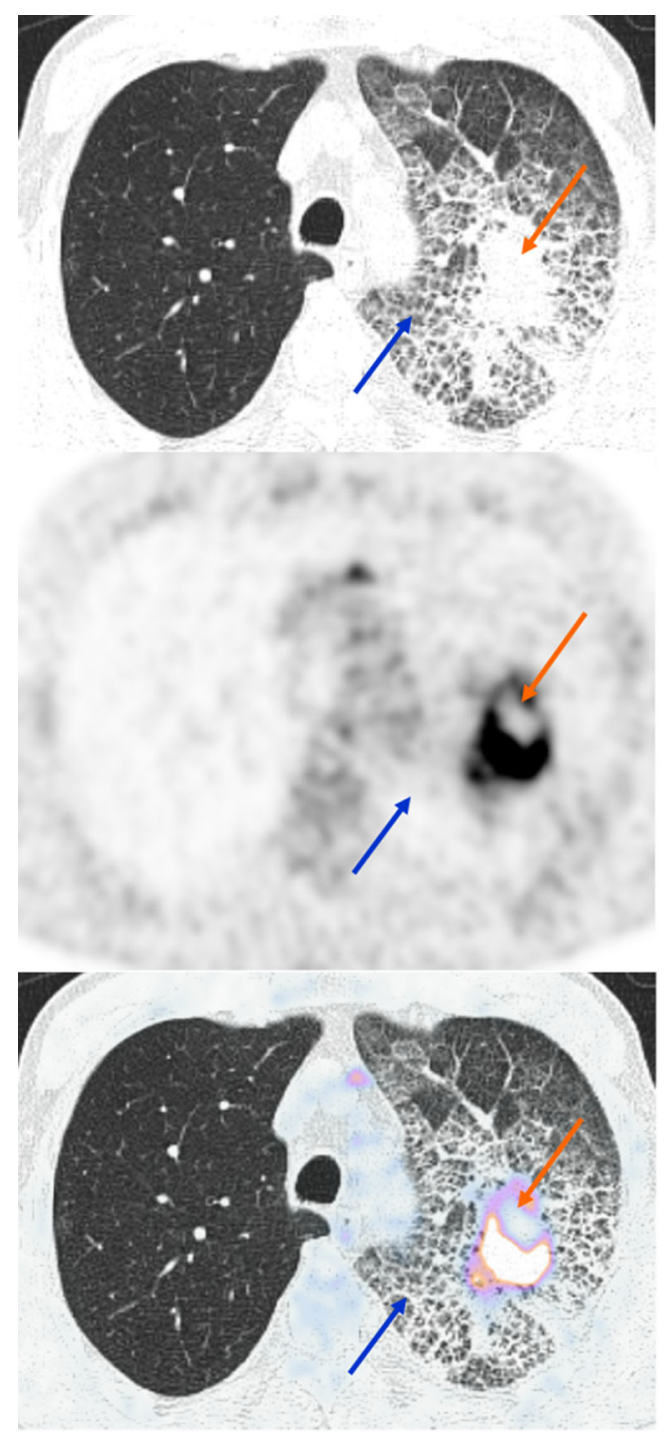

Fig. 5 18F-FDG PET/CT (axial CT-upper row, axial PET- middle row, axial fused PET/CT-lower row) showing increased FDG uptake of a lung mass (orange arrow) with minimal 18F-FDG uptake of GGO, interlobular septal thickening and intralobular lines mostly in the left upper lobe of the lung (blue arrow). SUVmax 15.75

\section{Conclusion}

Nuclear medicine physicians should familiarize themselves with the pattern of interstitial anomalies on lung CT images. Indeed, identifying asymptomatic infected patients is important, so that they can be treated efficiently and ensure the safety of caregivers and the public.
Funding This work has been supported in part by grants from the French National Agency for Research called "Investissements d'Avenir" IRON Labex $n^{\circ}$ ANR-11-LABX-0018-01 and ArronaxPlus Equipex n ${ }^{\circ}$ ANR11-EQPX-0004 and by grant from INCa-DGOS-Inserm_12558 (SIRIC ILIAD).

\section{Compliance with ethical standards}

Conflict of interest The authors declare that they have no conflict of interest.

Ethical approval All procedures performed in studies involving human participants were in accordance with the ethical standards of the institutional and national research committee and with the 1964 Helsinki declaration and its later amendments or comparable ethical standards. The study was approved by the locals Ethics Committee of the two institutes (Comité éthique du CHU de Nantes, Comité éthique de l'Institut de Cancérologie de l'Ouest René Gauducheau).

Informed consent Patients have been informed of access to their data and publication.

\section{References}

1. Guan $\mathrm{W}, \mathrm{Ni} \mathrm{Z}, \mathrm{Hu} \mathrm{Y}$, et al. Clinical characteristics of coronavirus disease 2019 in China. N Engl J Med. 2020;382(18):1708-20. https://doi.org/10.1056/NEJMoa2002032.

2. Chung M, Bernheim A, Mei X, et al. CT imaging features of 2019 novel coronavirus (2019-nCoV). Radiology. 2020;295(1):202-7. https://doi.org/10.1148/radiol.2020200230.

3. Inui S, Fujikawa A, Jitsu M, et al. Chest CT findings in cases from the cruise ship "diamond princess" with coronavirus disease 2019 (COVID-19). Radiol Cardiothorac Imaging. 2020;2(2):e200110. https://doi.org/10.1148/ryct.2020200110.

4. Shi H, Han X, Jiang N, et al. Radiological findings from 81 patients with COVID-19 pneumonia in Wuhan, China: a descriptive study. Lancet Infect Dis. 2020;20(4):425-34. https://doi.org/10.1016/ S1473-3099(20)30086-4.

5. Albano D, Bertagna F, Bertoli $\mathrm{M}$, et al. Incidental findings suggestive of COVID-19 in asymptomatic patients undergoing nuclear medicine procedures in a high-prevalence region. J Nucl Med. 2020;61(5):632-6. https://doi.org/10.2967/jnumed.120.246256.

6. Bernheim A, Mei X, Huang M, et al. Chest CT findings in coronavirus disease-19 (COVID-19): relationship to duration of infection. Radiology. 2020;295(3):200463. https://doi.org/10.1148/radiol. 2020200463.

7. Qin C, Liu F, Yen T-C, Lan X. 18F-FDG PET/CT findings of COVID-19: a series of four highly suspected cases. Eur J Nucl Med Mol Imaging. 2020;47(5):1281-6. https://doi.org/10.1007/ s00259-020-04734-w.

Publisher's note Springer Nature remains neutral with regard to jurisdictional claims in published maps and institutional affiliations. 